\title{
From the 5 Main Human Psychological Sufferings to Robots Human-Like Psychological Functioning
}

\author{
Francis Lavergne ${ }^{1 *}$ and Nicolas Marie $^{2}$ \\ ${ }^{1}$ Medical-Trial, 50 Boulevard Arago, 75013 Paris, France \\ ${ }^{2}$ Modal’X, Université Paris 10, 200 Avenue de la République, 92000 Nanterre, France
}

Received: January 30, 2018; Accepted: February 21, 2018; Published: March 07, 2018

*Corresponding author: Francis Lavergne, MD, Medical-Trial, 50 Boulevard Arago, 75013 Paris, France, Tel: $33-(0) 686780641$; E-mail: lavergne.francis100@gmail.com

\begin{abstract}
Introduction: Schemas represent a stable vision of oneself. Young's schema questionnaire-s3 (YSQ-s3) presents statements of 90 perceptions of oneself, specifying 18 Early Maladaptive Schemas (EMS). We studied the intensity and inter-relations of the 18 EMS and how they pool together into specific dimensions. In doing this research, 1) we want to identify the clusters, the dimensions that would explain most efficiently and economically, all the sufferings and beliefs presented by the patients. 2) We discuss the best understanding for the findings and proposed an interpretation based on Cognitive and Behavioral Therapy (CBT). 3) We speculate that the results and the CBT model could be used to program a "human-like psychology for robots"

Method: Two hundred and ninety four subjects (282 patients from a French medical practice in psychiatry and 12 volunteers) filled in a French version of the YSQ-s3. Item scores range from 1 to 6 . Only the scores of 4 "Moderately true of me" or higher were kept for the statistical analysis. Data analysis was conducted using descriptive statistics, principal component analysis, and hierarchical clustering analysis.

Result: The EMS mean scores ranged from 3.4 to 12.9 and standard deviations from 5.9 to 9.7. EMS score correlations range from 0.009 to 0.55. The principal component analysis, that provides linear combinations of each EMS score, yields only one meaningful component. Indeed, the screen plot that provides the Eigen values associated with each principal component, suggests keeping only the first component. This component presents a size-effect and represents the "global scores intensity". The hierarchical clustering analysis fits the 18 EMS in 5 clusters ( $\left.\mathrm{r}^{2}=0.4\right)$ : (1) "Avoidance" (with 3 EMS: emotional deprivation, social isolation/alienation, emotional inhibition), (2) "Give" (with 1 EMS: self-sacrifice), (3) "Take" (with 3 EMS: entitlement/grandiosity, insufficient self-control/self-discipline, approval-seeking/recognition-seeking) (4) "Awareness" (with 8 EMS: abandonment/instability, mistrust/abuse, defectiveness/shame, dependence/incompetence, vulnerability to harm or illness, enmeshment/ undeveloped self, failure, subjugation) (5) "Faith" (with 3 EMS: negativity/pessimism, unrelenting standards/hyper-criticalness, punitiveness). When the hierarchical clustering analysis is applied to the population $(n=294)$, it yields 6 classes of patients. The mean score of the 5 clusters can describe these classes.
\end{abstract}

Discussion: In our understanding the 5 clusters could fit the CBT model in which emotions and cognitions determine the subject's behavioral response. "Avoidance, Give, Take" represent 3 types of relationship to others (on a behavioral level). The dimension "Awareness" represents the fears and losses (on the emotional level) and "Faith" represents beliefs and consciousness (on the cognitive level).

On a psychological perspective this model can be useful to specify personality and clinical disorders in psychiatry. The 5 clusters seem closely related to the 5 sub-scales of the NEO-PI-R (a well established personality scale) and to 4 domains of DSM-5, criteria B for personality disorders (the ongoing research for personality disorder in the fifth version of the Diagnostic System Medical).

On a robotic perspective this model may be useful to design robots human-like psychological functioning.

keywords: Psychology; Schema therapy; Young schema questionnaire; YSQ-s3; Behavior; Robot psychology;

\section{Abbreviations}

YSQ-s3: Young schema questionnaire small form of version 3; EMS: Early Maladaptive Schema; CBT: Cognitive Behavioral Therapy; DSM-5: Diagnostic and Statistical Manual of Mental Disorders version 5.

\section{Introduction}

Wikipedia describes EMS as "self-defeating emotional and cognitive patterns established from childhood and repeated throughout life. They may be made up of emotional memories of past hurt, tragedy, fear, abuse, neglect, unmet safety needs, 
abandonment, or lack of normal human affection in general. EMS can also include bodily sensations associated with such emotional memories. EMS can have different levels of severity and pervasiveness: the more severe the schema, the more intense the negative emotion when the schema is triggered and the longer it lasts; the more pervasive the schema, the greater the number of situations that trigger it." This schema's pervasiveness characterizes personality disorders in which specific events induce systematic type of response. For example a frustrating situation induces systematically an anger response in narcissistic personality.

Jeffrey Young clinically organized the 18 schemas into 5 theoretical domains:

i) Disconnection/Rejection includes 5 schemas:

Abandonment/Instability

Mistrust/Abuse

Emotional Deprivation

Defectiveness/Shame

Social Isolation/Alienation

ii) Impaired Autonomy and/or Performance include 4 schemas:

Dependence/Incompetence

Vulnerability to Harm or Illness

Enmeshment/Undeveloped Self

Failure

iii) Impaired Limits includes 2 schemas:

Entitlement/Grandiosity

Insufficient Self-Control and/or Self-Discipline

iv) Other-Directedness includes 3 schemas:

Subjugation

Self-Sacrifice

Approval-Seeking/Recognition-Seeking

v) Overvigilance/Inhibition includes 4 schemas:

Negativity/Pessimism

Emotional Inhibition

Unrelenting Standards/Hypercriticalness

Punitiveness

We published, a statistical analysis of the Young Schema Questionnaire (YSQ-s3), in a french journal of psychiatry, l'Encéphale [1]. The methodology and results are given hereunder. Our interpretation of the results, in line with Cognitive and Behavioral Therapy (CBT) model proposes that the sufferings scored by the subject express a) memory of painful emotions, b) cognitive psycho-rigidity, c) excessive behavioral pervasiveness in 3 main dimensions. Excessive dominant "Take" behavior, Excessive dependent "Give" behavior, Excessive "Avoidance" behavior. This CBT model of the sufferings scored by patients can inspire the design of human-like psychological functioning for robots.

In order to identify the clusters of all the sufferings and beliefs presented by the patients, we analyze the YSQ-s3 in a clinical practice, with the methodology and the results described hereunder.

\section{Method}

\section{Population}

The population is made of 282 patients from my CBT practice in Paris and 12 healthy volunteers from my surroundings. Patients presented anxiety disorders, depression and personality disorders, excluding antisocial personality and excluding psychotic disorders. Volunteers and patients filled-in the YSQ-s3 at home.

\section{Assessment}

The assessment tool is the YSQ s-3. The items represent a large panel of statements about oneself: complains, judgments, sufferings. EMS is enduring inner representations and beliefs about oneself and others. The patients asses the intensity of each statement for himself. The 90 items of the questionnaire express feelings and mostly suffering about one's life. For example the first EMS is composed of 5 items:

1) "I haven't had someone to nurture me, share him/her with me, or care deeply about everything that happens to me".

2) I find myself clinging to people; I'm close to because I'm afraid they'll leave.

3) I find that people will take advantage of me.

4) I don't fit in.

5) No man/woman I desire could love me once he or she saw my defects of flaws.

The patient should recall, feel and compute the frequency and duration of the EMS items in his life. Each item intensity is scored from 1 to 6 .

1 = Completely untrue of me

$2=$ Mostly untrue of me

$3=$ Slightly more true than untrue

4 = Moderately true of me

$5=$ Mostly true of me

6 = Describes me perfectly

The EMS scores represent the patient's perceptions and statements about him-self and the scores intensity is a measure of the degree of invasiveness. The scores of 4, 5, 6 express a truth for the subject. Scores that range from 4 to 6 express long standing true personality trait and sufferings. This is what we are looking 
for when we are studying the main sufferings that characterize at best the subject's personality. We reported the 90 items scores on an excel sheet, changing the scores 1, 2, 3 into a "0" and keeping unchanged the scores of $4,5,6$. The EMS score is the sum of the 5 item scores. EMS scores range from 0 to 30 . The YSQ-s3 with 18 EMS cover different and meaningful psychological themes.

\section{Statistical Analysis}

Data analysis was conducted using descriptive statistics, principal component analysis and hierarchical clustering analysis with Statistica 7. Hierarchical clustering analysis was done with XLStat 7.5.2.

\section{Results}

We describe the mean and standard deviation of the 18 EMS scores; we looked at the in-between relationship of the EMSs and the way they pull together under 5 different dimensions. We looked at the in-between relationship of the 5 dimensions. We also analyze the population with a hierarchical clustering analysis and found 6 classes of patients. We finally measured the 5 dimension scores in the 6 classes of patients and tried to interpret the classes from dimension's characteristics.

\section{EMS Descriptive Statistics}

The Figure 1 gives the means and standard deviations of each EMS. The mean scores of the 18 EMS vary from 3.42 points for "dependency" to 12.98 points for "unrelenting standards" and the standard deviations vary from 5.91 for "dependency to 9.72 for "negativity / pessimism".
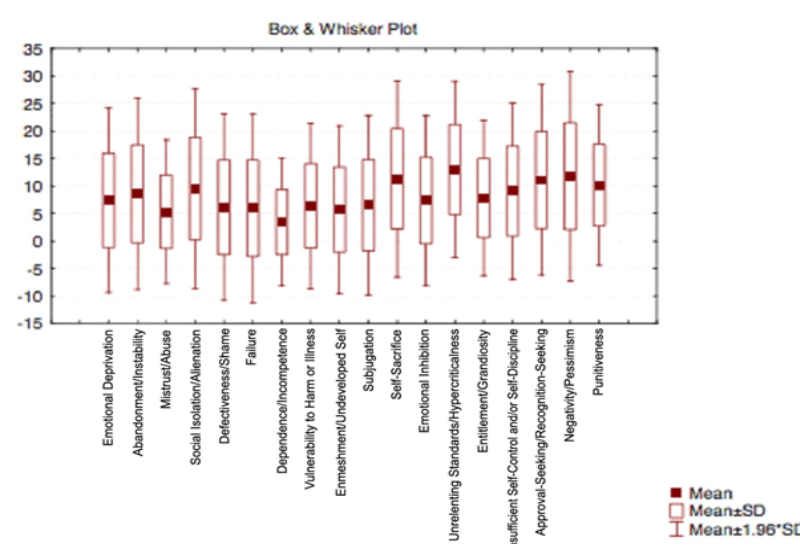

Figure 1: EMS mean + S.D

90 YSQ-s3 item scores are given by the patients on the questionnaire. The scores of 1,2,3 are deleted (value=0), scores of 4,5,6 are kept unchanged. EMS score is the sum of five items.

\section{EMS correlations}

The 18 EMSs are inter-related. 2 by 2 correlations yields a Pearson's coefficients that vary from $r=0.009$ to $r=0.55$. "mistrust/abuse" is the only EMS correlated to all EMSs. Self Sacrifice is the less often correlated EMS $(n=6)$. These, rather weak correlations express the quality of the questionnaire in which all EMSs differ sufficiently from one another and all bring a specific contribution. EMSs are not redundant.

\section{EMS clustering into dimensions}

With the hierarchical clustering analysis, the 18 EMSs cluster into 5 dimensions, when one follows the best statistical fit $(\mathrm{r} 2=0.40)$. The best fit is characterized by longer branches at the step 14 of the hierarchical clustering analysis algorithm. This result is the only one that we discuss and use for our "human-like psychology for robots" speculation (Figure 2).

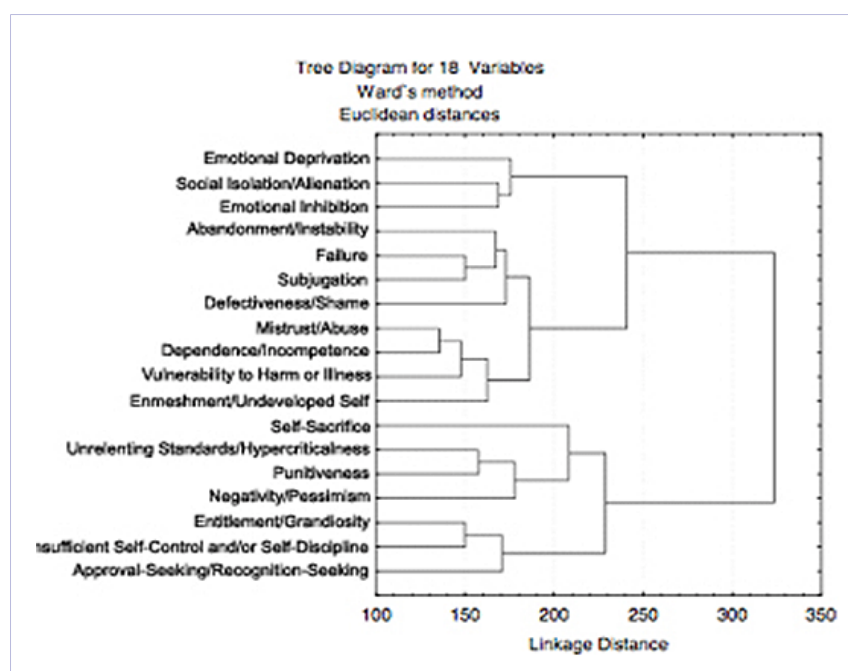

Figure 2: Hierarchical Clustering Analysis of the EMS $(n=18)$, best statistical fit determines 5 clusters

These 5 clusters are named "Awareness, Faith, Give, Take, and Avoidance".

"Awareness" is made of 8 EMSs: abandonment/instability, mistrust/abuse, defectiveness/shame, failure to achieve, dependence/incompetence, vulnerability to harm or illness, enmeshment/under-developed self, subjugation.

"Faith" is made of 3 EMSs: Unrelenting standards, Negativity/ Pessimism, Punitiveness.

"Take" is made of 3 EMSs: Entitlement/Grandiosity, Insufficient self-control, Approval-seeking.

"Give" is made of only one EMS: Self-sacrifice.

"Avoidance" is made of 3 EMSs: Emotional deprivation, Social isolation/Alienation, Emotional inhibition.

\section{Cluster's correlations are given in table 1}

Correlations (Sheet 1 in Matrice Patients $\mathrm{x}$ Domaines), marked correlations (in red) are significant at $\mathrm{p}<0.05, \mathrm{n}=294$ (Casewise deletion of missing data) (Table 1)

All the 5 clusters are 2 by 2 inter related with 2 exceptions. The 2 exceptions are "Give"/"Avoidance" ( $r=0.07)$, and "Give" / Take" $(\mathrm{r}=0.10)$. The "Give" cluster is independent of the "Take" and "Avoidance" cluster. "Awareness" and "Faith" present the highest correlation: $\mathrm{r}=0.551$. 
Table 1: Correlation matrice of the 5 dimensions (clusters: "Awareness”, “Faith”, Take”, “Give”, "Avoidance”) marked correlations in red are significant at $\mathrm{p}<0.05$

\begin{tabular}{|c|c|c|c|c|c|}
\hline & “Awareness" & “Faith” & “Take” & “Give” & “Avoidance” \\
\hline "Awareness" & 1 & 0.551 & 0.504 & 0.364 & 0.434 \\
\hline “Faith" & - & 1 & 0.403 & 0.222 & 0.358 \\
\hline “Take" & - & - & 1 & 0.108 & 0.269 \\
\hline “Give" & - & - & - & 1 & 0.07 \\
\hline “Avoidance" & - & - & - & - & 1 \\
\hline
\end{tabular}

\section{Patient's hierarchical clustering analysis}

The 294 patients and non patients cluster into 6 classes, when one follows the best statistical fit, in a hierarchical clustering analysis. These results concern human diversity and pathology; they do not concern the construction of "human-like psychology" (Figure 3).

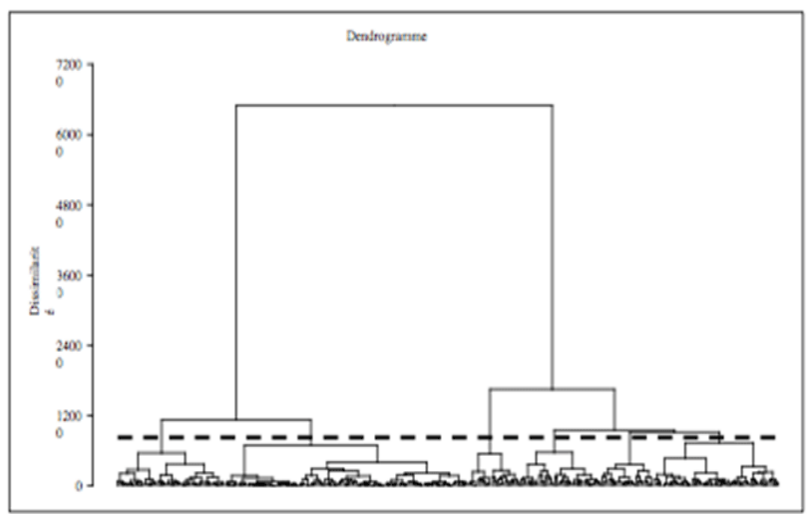

Figure 3: Hierarchical Clustering Analysis of the population ( $\mathrm{n}=294)$, best statistical fit determines 6 clusters

\section{Mean scores of the 5 clusters for each class of patients}

Dimensions scores range from 3.0 to 20.6 points for the 5 clusters, across the 6 classes. (Table 2)

The scores of the 5 clusters are obviously related to the global score (sum of 18 EMS' scores). This relation is extremely tight for "Awareness" ( $r=0.91)$ and "Faith" ( $r=0.90)$.

A reading of these results, suggest that a "human-like functioning" could yields 6 different psychological classes of robots. A psychiatric reading of these scores tentatively proposes that class 1 points toward schizoid and avoidance personality disorders with a predominant "Give and Avoidance" cluster (scores $>11$ ), class 2 is the "healthier" category with low scores except for the "Give" cluster (score $>10$ ), class 3 is the sicker, and confused patients that points toward borderline and possibly schizotypic personality disorders with high "Awareness and Faith" (scores>16) and equal "Take, Give, Avoidance" dimensions (scores $>14$ and $<17$ ), class 4 points toward the dependence personality disorder with a high "Give" cluster (score>20), class 5 points toward obsessional and paranoiac personality disorders with high "Faith and "Avoidance" cluster (scores $>17$ ), class 6 points toward histrionic, narcissistic and possibly antisocial personality with a high "Take" cluster (score>13).

Table 2: Scores of the 5 dimensions (clusters) and average score of the 5 dimensions, in the 6 classes of patients.

Correlations of the 5 dimensions (clusters: "Awareness", "Faith", Take”, "Give”, "Avoidance") with the global score of the questionnaire YSQ-s3

\begin{tabular}{|c|c|c|c|c|c|c|}
\hline & “Awareness" & “Faith” & “Take” & “Give” & “Avoidance” & $\begin{array}{c}\mathbf{5} \text { dimensions } \\
\text { Average }\end{array}$ \\
\hline Class 1 & 3,54 & 10,69 & 6,64 & 12,40 & 11,53 & 8,96 \\
\hline Class 2 & 2,23 & 7,39 & 5,26 & 10,14 & 3,04 & 5,61 \\
\hline Class 3 & 16,39 & 16,22 & 16,48 & 14,39 & 14,75 & 15,65 \\
\hline Class 4 & 10,05 & 15,40 & 11,23 & 20,64 & 9,87 & 13,44 \\
\hline Class 5 & 7,77 & 17,70 & 13,48 & 8,09 & 17,23 & 12,85 \\
\hline Class 6 & 7,82 & 13,90 & 13,65 & 6,70 & 7,34 & 9,88 \\
\hline $\begin{array}{c}\text { Correlation to global } \\
\text { score }\end{array}$ & $\mathrm{R}=0,918$ & $\mathrm{R}=0.908$ & $\mathrm{R}=0,854$ & $\mathrm{R}=0,439$ & $\mathrm{R}=0,782$ & \\
\hline
\end{tabular}

\section{Discussion}

\section{We discuss the best understanding for the findings and proposed an interpretation based on CBT}

Do the 5 clusters fit with the postulated "Unsatisfied Infantile Needs" proposed by J. Young? if not, how can we understand these 5 clusters? How do they compare to well establish personality models? What does the EMS literature tells us about diagnosis, treatments and mood?

\section{Unsatisfied infantile needs}

Jeffrey Young postulate 5 psychological domains of "Unsatisfied Infantile Needs". In theory, EMSs develop when the basic infantile needs are unmet. It would be coherent that all EMSs cluster into the five "Unsatisfied infantile needs" domains, but we cannot confirm this idea. Only 2 EMSs out of 3 are correctly classified in the postulated "Unsatisfied Infantile Need" domains. We, as Sakulsriprasert C, et al. [2] and Kriston L, et al. [3] fail to support Young's theoretical domains. 


\section{Interpretation of the 5 clusters in a CBT model}

We understand the sufferings inside the CBT model: stimulation induces a response, a behavior, through an emotion and cognition synergetic interaction. Inside this model emotions would be expressed by the dimension "Awareness", the cognition would be expressed by the dimension "Faith" and the three main dysfunctional responses at the behavioral level would be expressed by "Take" too-much, "Give" too-much and too much "Avoidance". At the emotional level, the cluster "Awareness" could represent the negative emotions (disgust, hostility, fear, depression). In a clinical setting, the most common negative emotions are anxiety and depression. At the cognitive level, the cluster "Faith" is not only the cognitions related to the emotions but also the proximity of the cognitions to the emotions. "Faith" is the trust we have in our point of view and feelings; it is our roots and our beliefs. Understanding that emotion gives the sense of truth, closer your "Faith" is to your emotions, the stronger you believe. "Faith" is a dimension that holds the person together and resists changing. With "Faith" the person trusts his point of view.

These two clusters, "Awareness" and "Faith" could be seen as the 2 pressures exerted upon the patient. "Awareness" is the body's perceptions and emotions, "Faith" is the mental perception of oneself truth. Both, "Awareness" and "Faith" have extremely high correlation with YSQ-s3 global score ( $r>0.90)$. The negative emotions described in the "Awareness" cluster and the psycho-rigidity described in the "Faith" cluster are the actors of the patient's sufferings.

Then the sufferings are characterized at a behavioral level. Behaviors can be characterized in the "Take", "Give", "Avoidance" clusters. We discuss later the relations between these 3 behavioral clusters and other personality disorders models such the NEOPI and the DSM-5. Toxicity appears with high EMSs scores. All classes of patients, except the healthier class present a strong correlation with the "Faith" cluster. In clinic, excessive "Faith" + "Give"= Generosity toxicity, "Faith"+ "Take"= Grandiosity toxicity, "Faith"+ "Avoidance"= Psycho-rigidity toxicity

\section{YSQ-s3 comparison to other models of personality disorders}

How do the 5 clusters relate to the "big five" personality inventory (NEO-PI). Despite the fact that the "big five" studies personality traits and the EMS studies pathological personality traits, it is intuitive that the 5 main psychological sufferings and the big five personality inventory correspond to similar entities. 1) The "Awareness" cluster with its 8 EMS seems close, in meaning, to the NEO-PI sub-scale "Neuroticism" 2) the "Faith" cluster with its EMSs seems to be the extreme of NEO-PI subscale "Conscientiousness" and express the subject's strength in his values. 3) The "Take" cluster is like the extreme of the subscale "Extraversion", and represents a dominant behavior, 4) the "Give" cluster, is like the extreme of the sub-scale "Agreeableness", both are made of accepting behaviors. 5) the "Avoidance" cluster is very much like the negative polarity of the sub-scale "Openness to Experience". Studies from the literature show that EMSs have significant relations with NEO-PI sub-scales. Thimm JC, [4] finds substantial overlap between EMSs and neuroticism sub-scale in particular. While looking at perfectionism, Maloney, et al. [5] find that the sub-scale "Neuroticism" of the NEO-PI correlates with the first five EMSs of the YSQ-s3 ( $\mathrm{r}$ ranges from 0.258 to 0.562). Other EMSs have not been tested. Bahrami and Bahramizadeh, [6] found that EMSs from the clusters "Awareness", "Faith",

Table 3: Correlations between the 18 EMS and DSM-5 facets and dimensions, from Bach et all study (ref 7). The correlation presented in the table is the highest correlation ( $\mathrm{r}$ Max) the EMS presents with the DSM-5 facets and dimensions in the publication's data (ref 7).

\begin{tabular}{|c|c|c|c|c|c|}
\hline 5 main sufferings & EMS & r Max & DSM-5 facets & r Max & DSM-5 dimension \\
\hline & AB: Abandonment & .76 & Separation Insecurity & .81 & Negative Affectivity \\
\hline & MA: Mistrust / Abuse & .87 & Suspiciousness & .68 & Negative Affectivity \\
\hline & DS: Defectiveness / Shame & .82 & Depressivity & .75 & Detachment \\
\hline & FA: Failure to archive & .74 & Depressivity & .64 & Detachment \\
\hline & DI: Dependance / Incompet. & .71 & Depressivity & .66 & Negative Affectivity \\
\hline & VH: Vulnerability to Harm & .75 & Anxiousness & .70 & Negative Affectivity \\
\hline & EM: Enmeshment & .58 & Anxiousness & .62 & Negative Affectivity \\
\hline Awareness & SB: Subjugation & .71 & Depressivity & .67 & Negative Affectivity \\
\hline \multirow[t]{3}{*}{ Faith } & US: Unrelenting standards & .63 & Rigid perfectionism & .44 & Negative Aff /Detachment \\
\hline & PN: Pessimism / Negativity & .82 & Anxiousness & .76 & Negative Affectivity \\
\hline & SP: Self Punitiveness & .66 & Depressivity & .59 & Detachment \\
\hline \multirow{2}{*}{ Take } & ET: Entitlement & .64 & Grandiosity & .63 & Antagonism \\
\hline & AS: Approval seeking & .51 & Attention Seeking & .49 & Negative Affectivity \\
\hline Give & SS: Self sacrifice & .42 & Suspiciousness & .42 & Negative Affectivity \\
\hline \multirow[b]{3}{*}{ Avoidance } & ED: Emotional Deprivation & .66 & Depressivity & .65 & Detachment \\
\hline & SI: Social Isolation & .80 & Depressivity & .76 & Detachment \\
\hline & EI: Emotional Inhibition & .71 & Withdrawal & .73 & Detachment \\
\hline
\end{tabular}


"Avoidance" are negative predictors of "Agreeableness" like an anxious body, a rigid mind and an avoiding behavior are opposite to "Agreeableness".

How does the 5 clusters relate to the Diagnostic and Statistical Manual of Mental Disorders (DSM-5)? In the actual DSM-5 research, the criterium $\mathrm{B}$, with its 5 trait domains seems closely related to the 5 clusters, except for Psychoticism. Psychoticism with its wide range of culturally incongruent odd, behaviors and cognitions does not resemble any of the 5 clusters. Bach, et al. [7], in the Journal of Personality Disorders 2015, studies "How do DSM-5 personality traits align with schema therapy constructs". They find that the EMSs are strongly related to DSM-5 personality trait facets and domains. The Table 3 gives the DSM- 5 facets and domains most strongly correlated to the 18 EMSs. From Bach's data, we present the highest correlation ( $r$ Max) between the EMSs and the DSM-5 facets (range from 0.42 to 0.87 ) and between the EMSs and DSM-5 domains (range from 0.42 to 0.81 ) in their study (Table 3).

The 8 EMSs from the "Awareness" cluster are related to the "Negative Affectivity" ( $\mathrm{n}=6 / 8, \mathrm{r}$ from 0.62 to 0.81 ) and to the "Detachment" dimensions $(n=2 / 8, r$ ranges from 0.64 to $0.75)$. This is in line with the negative emotions of fear and loss, characteristic of "Awareness" in our study. "Awareness" covers both clinical anxiety and depression in the DSM-5.

The 3 EMSs of "Faith" relate equally to the "Negative Affectivity" and "Detachment" dimensions (range $r=0.44$ to 0.76 ). The "Faith" cluster relates to rigid perfectionism, anxiousness and depressivity facets.

The 3 behavioral clusters relate to DSM- 5 criteria B.

- The 3 EMSs from "Take" relate to 3 different DSM-5 domains: "Antagonism" ( $n=1 / 3, r=0.63)$, "Disinhibition" ( $n=1 / 3, r=0.75)$ and "Negative Affectivity" ( $n=1 / 3, r=0.49)$. "Take" EMSs could describe a patient with a high ego ( $\mathrm{r}=0.64$ with Grandiosity facet) but unstable and vulnerable ( $\mathrm{r}=0.74$ with Distractibility facet and $r=0.51$ with Attention seeking facet).

-The only EMS from the "Give" cluster relates to the "Negative Affectivity" domain ( $\mathrm{r}=0.42$ ) and to "Suspiciousness" facet ( $r=0.42$ ). "Give" is related to anxious feelings. From all 18 EMSs of the YSQ-s3, "Self-Sacrifice" presents the lowest "highest" correlation with DSM-5 facets $(r=0.42)$ and domains ( $r=0.42)$. The "Give" dimension correlates poorly (ranges from 0.03 to 0.42 ) to DSM-5 facets. It could be because the "Give" dimension with its acceptation, humbleness and generosity is like social glue, partly protective from social sufferings, therapeutic by himself and not clinically meaningful. In the Encéphale 2015 study, EMS self-sacrifice was noted as the least toxic of all EMSs because it was the less contributive to the global score and because the "Give" cluster was high in the healthier class of patients with the lower global score. The low correlations between the "Give" cluster and the DSM-5 facets and domaines could be due to the absence of DSM-5 item expressing pathological generosity. The "Give" dimension, necessary for dependence personality disorder diagnosis is lacking in DSM-5 criteria B.
- The 3 EMSs from the "Avoidance" cluster relate tightly to the "Detachment" domain ( $n=3 / 3$ range $r=$ from 0.65 to 0.76$)$. These EMSs relate to the facet "withdrawal" ( $\mathrm{r}=0.71)$ and twice to the facet "Depressivity" ( $\mathrm{r}=0.66$ and $\mathrm{r}=0.80$ ). "Avoidance" behavior is related to isolation and depressive mood.

No EMS has its highest correlation with DSM-5 "Psychoticism" facets or domaine. YSQ-s3 could be missing the DSM-5 "Psychoticism" trait and in clinic could be missing the schizotypic personality disorder. An alternative interpretation would be that the schizotypic personality disorder is expressed by the confusion arising from equal levels of the "Take and Give" clusters.

\section{The EMSs in clinical research: diagnostic, therapy, mood}

Clinically, Jeffrey Young appreciates that an EMS score higher than 9 points starts to be invasive in the patient's life [8]. Studies, with different versions of the YSQ, show that total score and EMS scores are related to pathology and disease intensity. With bulimia nervosa patients, Unoka, et al. [9] finds that the Body Mass Index presents a negative $(\mathrm{p}<0.01)$ relationship to EMSs "defectiveness, failure, dependence, enmeshments, subjugation, approval-seeking". With depression, on the Beck Depression Inventory scale, Cormier, et al. [10] finds that EMSs scores increase with disease intensity: in moderately depressed participants 6 EMSs are increased, while in severely depressed participants 15 EMSs are increased compared to control. In chronically traumatized patients seeking outpatient psychiatric treatment, Dutra, et al. [11] finds that suicide risk variables were most highly correlated with 3 EMSs: social isolation/alienation, defectiveness/shame and failure. In borderline personality disorder, Mauchand, et al. [12] shows that total YSQ scores differs $(p<0.001)$ between borderline personality disorder and control group. Total YSQ-s3 score is clearly an indication of psychic suffering and psychic suffering should obviously characterize many diseases in psychiatry. The YSQ global score is related to disease severity. Thiel, et al. [13] show that obsessive-compulsive patients, none responding to CBT have higher global YSQ-s3 score at entry than responding patients. Resistance to treatment is a sign of disease severity and predicts high YSQ score. In contrast Wegener and Leppanen studies show that psychiatric treatments reduce EMS scores. Wegener, et al. [14], shows that symptoms reduction of depression after treatment is strongly associated with a score reduction in autonomy and performance EMSs. Leppanen, et al. [15] show, in borderline personality disorder $(n=18)$, that EMSs scores were reduced after one year of schemafocused therapy and dialectical behavioral therapy compared to treatment as usual $(n=27)$. The combined treatment model shows a statistically significant reduction in 8 out of 18 EMS, while patients receiving treatment as usual did not demonstrate any significant change in EMS scores. The YSQ total score increases with disease severity and decreases with efficacious treatments.

EMSs are also sensitive to mood induction: 30 participants completed the YSQ on three different occasions: in neutral mood, following happy and depressed mood inductions. Stopa and Waters, [16] find that EMS "emotional deprivation" and 
"defectiveness" scores increase after the depressed mood induction, whereas "entitlement" scores increase after the happy mood induction. It is impressive that a depressive mood induction increases the EMSs corresponding to the "Awareness and Avoidance" dimension and that a happy mood induction increases the "Take" dimension.

In summary, researches show that EMS scores are sensitive to pathology, to treatment and to mood. The 5 clusters are compatible with the NEO-PI dimensions and closely related to 4 dimensions of the DSM-5, criteria B dimensions.

\section{We speculate that the 5 clusters in the CBT model could be used to program a "human-like psychology for robots" From sufferings to health}

The YSQ-s3 questionnaire is very meaningful clinically, it list all the sufferings usually seen in practice, like shame, arrogance, self-sacrifice, but it lacks the healthy part in the patients. Excessive "Faith" is, in the analysis we made in the Encéphale journal, the most toxic cluster since it is toxic (score $>9$ ) in all groups of patients with the exception of class 2 patients, which is the most healthy group. Nevertheless there is a positive side of "Faith", in religious belief and optimism. "Faith" can be related to positive or negative emotions with different intensities. Positive emotions and cognitions are needed to describe healthy behaviors and "human-like psychology" for robots.

Could we introduce a healthy dimension in the behaviors, in order to represent the healthy side that is not studied in the YSQ-s3? Our answer is no. "Take" and "Give" are obviously a form of opposition, and it would be elegant to characterize the behaviors in terms of 2 polarities. The "Take / Give" polarity and the "GO / Avoidance" polarity. The "GO" dimension could be the opposite of "Avoidance" and would represent desires, motivations and gains. It would be elegant but misleading. In our CBT model, the psychic life is made from the interaction of emotions and cognitions, as neurologist, Antonio Damasio proposes. The healthy part is made from positive emotions and cognitive openness, (the opposite of psycho-rigidity), they is no need to change the 3 behavioral clusters. They are appropriate even if they were selected from our understanding of CBT.

The "Go" response, in a fearful condition would be inappropriate. In a fearful situation the animal does not explore calmly the surrounding. We cannot add a Go dimension only because the YSQ-s3 does not explore the healthy personality or because it is elegant.

The "Take versus Give versus Avoidance" represents a "surface behavior" on which all behaviors could be represented. Under a fearful condition the rat can freeze, fly, or fight. Freeze would be an "avoidance" response, fly would be a submissive "Give" response, and fight would be a dominant "Take" response. The possible rat's reaction under stress is of 3 kinds, even if, in practice the rat usually freezes or flight and rarely fights. In fact, it must be a matter of cognition. Under a fearful situation and without a way out, it is likely that the rat computes that fight is a better option than freeze or flight. It is intriguing to see the movements of these 3 behaviors. Fight goes forward, flight goes backward and freeze does not move at all. Could that means that all behaviors are basically made of only 3 meta-actions? It seems that mechanically they are basically only 3 behavioral actions: forward, backward, equilibrium. This would be appropriate for all emotions. Love and desire usually have a forward behavior but in some case, the subject computes that backward is a better strategy. Even the neuron has 3 positions: depolarization, hyperpolarization and equilibrium. Even the synapses connectivity are in 3 directions: Long term Depression, Long Term Potentiating and equilibrium. Fundamentally we propose that the 3 behavioral clusters represent the well known "Take"/"Give" opposition (dominance versus submission) to which our results, added an "Avoidance" dimension. This dimension says that they will be no "Take" or "Give" if I do not engage in the relation. As observed by clinicians "Avoidance" is very powerful behavior. By refusing the interactions, the subject keeps the power, at the cost of losing the human link.

In summary, the analysis of the 5 clusters in the CBT model, from a population that suffers psychologically could be generalized to the healthy population. In the healthy population the emotions ("Awareness" cluster) would be more positive and the cognition ("Faith" cluster) would be less psycho-rigid, but the behaviors can still be represented on a surface behavior made from a triangle, with at the angles "Awareness", "Faith", "Avoidance".

\section{For a robotic human-like psychological functioning}

Do these data and their interpretations allow building a model for human-like psychological functioning in robots? We propose 2 rules for doing so.

\section{a) The CBT rule}

The CBT model is a scientific approach in psychology that could tentatively by apply to develop human-like psychologic functioning in robots. Intuitively we propose to translate the CBT equation: "Emotion + Cognition = Behavior", into a program. The CBT model proposes that the synergy of emotion and cognition produces the behavior. Psychic life is made of emotion in synergy with the cognition, like in our dreams. When one wakes up from his dream he can recall/compute a story, which represents the cognition and can recall/feel the emotions in his dream that represent his dream colors and emotions. From this understanding of the psychic life, the robotic model should have two components: the "Emotion" data base component and the "Faith" uncertainty component to compute a behavioral response. The "Emotion" data base could be made of memories of experiences. The experiences would present an event with its connection to an emotion (e.g.: the event of a smiling face is $95 \%$ connected to the emotion of pleasure). Emotions have quality and quantity. The "Faith" uncertainty component would compute the "adhesion value" of the experiences, the degree of pervasiveness of the emotion. This would compute, in the data base, the frequency of a smiling face in connection to pleasure and other emotions. Obviously the system must be able to learn by himself, to learn from its mistakes and to enhance his data base by experiences. 


\section{b) The "behavioral surface"}

Our research and interpretations from the Young schema questionnaire organizes human behaviors on a "surface behavior". The surface is made of a triangle with 3 summits: "Take", "Give", "Avoidance". The emotion-cognition synergy computes the robot's response on the "behavioral surface". The behavioral surface goes two ways. It is the interface, like our behaviors, with the world. It serves to decide my action and it serves to analyze the actions of others. My action can be pin-pointed on the surface behavior and the actions from others are also characterized by a point on the surface behavior.

\section{Conclusion}

The analysis of psychological suffering with the YSQ-s3 allows an interpretation inside the CBT model. The 5 clusters could represent the patient's emotion, cognition and 3 excessive behavioral responses: "Take, Give, Avoidance". This interpretation differs from other personality disorders scales and questionnaires that usually represent all personality traits at the same level. It can be criticized and discussed, but it sounds clinically meaningful and in accordance with the psychic life described as an interaction between emotions and cognitions.

We expect, from a program that would use our "surface behavior" to be able to detect a player's personality traits during a game of go and to adapt to all situations with a "human-like psychological functioning".

\section{References}

1. Lavergne F, Marie N, Mehran F. The 5 mental dimensions identified in early maladaptive schemas statistical analysis of Young's schema questionnaire (YSQ-s3)]. Encephale. 2015;41(4):314-322. doi 10.1016/j.encep.2014.08.005

2. Sakulsriprasert C, Phukao D, Kanjanawong S, Meemon N. The reliability and factor structure of Thai Young Schema Questionnaire-Short Form 3. Asian J Psychiatr. 2016;24:85-90.

3. Kriston L, Schäfer J, von Wolff A, Härter M, Hölzel LP. The latent factor structure of Young's early maladaptive schemas: are schemas organized into domains? J Clin Psychol. 2012;68(6):684-698. doi: $10.1002 /$ jclp. 21846

4. Thimm JC. Personality and early maladaptive schemas: a five-factor model perspective. J Behav Ther Exp Psychiatry. 2010;41(4):373-380. doi: 10.1016/j.jbtep.2010.03.009
5. Maloney GK, Egan SJ, Kane RT, Rees CS. An etiological model of perfectionism. PLoS One. 2014;9(5):e94757. doi: 10.1371/journal. pone.0094757

6. Bahrami Ehsan H, Bahramizadeh H. Early maladaptive schemas and agreeableness in personality five factor model. 2nd world conference on psychology, counselling and guidance 2011. Procedia - Social and Behavioral Sciences. 2011;30:547-551. doi:10.1016/j. sbspro.2011.10.107

7. Bach B, Lee C, Mortensen EL, Simonsen E. How Do DSM-5 Personality Traits Align With Schema Therapy Constructs? J Pers Disord. 2016;30(4):502-529. doi: 10.1521/pedi_2015_29_212

8. Young JE, Klosko JS, Weishaar ME. Schema therapy. New York: The Guilford Press; 2003.

9. Unoka Z, Tölgyes T, Czobor P. Early maladaptive schemas and body mass index in subgroups of eating disorders: a differential association. Compr Psychiatry. 2007;48(2):199-204.

10. Cormier A, Jourda B, Laros C, Walburg V, Callahan S. Influence between early maladaptive schemas and depression. Encephale. 2011;37(4):293-298. doi: 10.1016/j.encep.2011.01.001

11. Dutra L, Callahan K, Forman E, Mendelsohn M, Herman J. Core schemas and suicidality in a chronically traumatized population. J Nerv Ment Dis. 2008;196(1):71-74. doi: 10.1097/NMD.0b013e31815fa4c1

12. Mauchand P, Lachenal-Chevallet $\mathrm{K}$, Cottraux J. Validation empirique du questionnaire abrégé des schémas de Young (YSQ-s2) sur une population de patients présentant un trouble de personnalité borderline et de sujets témoins. L’Encéphale. 2011;37(2):138-143.

13.Thiel N, Tuschen-Caffier B, Herbst N, Külz AK, Nissen C, Hertenstein $\mathrm{E}$, et al. The prediction of treatment outcomes by early maladaptive schemas and schema modes in obsessive-compulsive disorder. BMC Psychiatry. 2014;14:362. doi: 10.1186/s12888-014-0362-0

14. Wegener I, Alfter S, Geiser F, Liedtke R, Conrad R. Schema change without schema therapy: the role of early maladaptive schemata for a successful treatment of major depression. Psychiatry. 2013;76(1):117. doi: $10.1521 /$ psyc.2013.76.1.1

15.Leppänen , Kärki A, Saariaho T, Lindeman S, Hakko H. Changes in schemas of patients with severe borderline personality disorder: the Oulu BPD study. Scand J Psychol. 2015;56(1):78-85. doi: 10.1111/ sjop.12172

16. Stopa L, Waters A. The effect of mood on responses to the Young Schema Questionnaire: short form. Psychol Psychother. 2005;78(Pt 1):45-57. 